\title{
First report of tetracycline-resistant Aeromonas veronii infection in Amur catfish (Silurus asotus) cultured in Korea
}

\author{
Hyo Yeon Kim ${ }^{1,2, \uparrow}$, Jin Woo Jun ${ }^{3, \dagger}$, Se Ra Lim ${ }^{1}$, Seon Young Park ${ }^{1}$, Jee Eun Han ${ }^{4}$, \\ Se Chang Park ${ }^{2, *}$, Ji Hyung Kim ${ }^{1, *}$ \\ ${ }^{1}$ Infectious Disease Research Center, Korea Research Institute of Bioscience and Biotechnology, Daejeon 34141, Korea \\ ${ }^{2}$ Laboratory of Aquatic Biomedicine, Research Institute for Veterinary Science, College of Veterinary Medicine, \\ Seoul National University, Seoul 08826, Korea \\ ${ }^{3}$ Department of Aquaculture, Korea National College of Agriculture and Fisheries, Jeonju 54874, Korea \\ ${ }^{4}$ Laboratory of Aquatic Biomedicine, College of Veterinary Medicine, Kyungpook National University, Daegu 41566, Korea
}

\begin{abstract}
Mass mortality in commercially cultured Amur catfish (Silurus asotus), showing symptoms of dermal ulcerations, occurred on a private farm in Mar 2019 in Korea. $\beta$-hemolytic bacteria were isolated from the ulcers and kidneys of the fish and identified as Aeromonas veronii. The isolate was resistant to tetracycline and possessed cytotoxic heat-labile enterotoxin (aerolysin/ hemolysin). We investigated the genetic determinants associated with tetracycline resistance, and the isolate has been confirmed to simultaneously possess tet $A$ and tet $E$ genes. This is the first report on the occurrence of tetracycline-resistant $A$. veronii infection related to mass mortality in commercially cultured Amur catfish in Korea.
\end{abstract}

Keywords: Amur catfish (Silurus asotus), Aeromonas, tetracycline resistance, tetA, tetE

*Corresponding author
Ji Hyung Kim
Infectious Disease Research Center, Korea
Research Institute of Bioscience and Biotech-
nology, 125 Gwahak-ro, Yuseong-gu, Daejeon
34141, Korea
Tel: +82-42-879-8272
Fax: +82-42-879-8498
E-mail: kzh81@kribb.re.kr
Se Chang Park
Laboratory of Aquatic Biomedicine, Research
Institute for Veterinary Science, College of Vet-
erinary Medicine, Seoul National University, 1
Gwanak-ro, Gwanak-gu, Seoul 08826, Korea
Tel: +82-2-880-1282
Fax: +82-2-880-1213
E-mail: parksec@snu.ac.kr
†The first two authors contributed equally to
this work.
ORCID
Hyo Yeon Kim
https://orcid.org/0000-0002-3507-0311
Jin Woo Jun
https://orcid.org/0000-0002-0318-6889
Se Ra Lim
https://orcid.org/0000-0002-8915-3876
Seon Young Park
https://orcid.org/0000-0002-1351-7557
Jee Eun Han
https://orcid.org/0000-0002-2905-7524
Ji Hyung Kim
https://orcid.org/0000-0002-7921-2625
Se Chang Park
https://orcid.org/0000-0001-9821-387X
Conflict of Interest
The authors declare no conflicts of interest.
Received: July 25, 2019
Revised: September 9, 2019
Accepted: October 4, 2019
thed

\section{*Corresponding author}

Research Institute of Bioscience and Biotechnology, 125 Gwahak-ro, Yuseong-gu, Daejeon 34141, Korea

Tel: $+82-42-879-8272$

Fax: +82-42-879-8498

E-mail:kzh81@kribb.re.kr

Se Chang Park

Institute for Veter erinary Medicine, Seoul National University, 1 Gwanak-ro, Gwanak-gu, Seoul 08826, Korea

Tel: +82-2-880-1282

E-mail: parksec@snu.ac.kr

The first two authors contributed equally to this work

https://orcid.org/0000-0002-3507-0311

Jin Woo Jun

Se Ra Lim

https://orcid.org/0000-0002-8915-3876

Seon Young Park

Jee Eun Han

https://orcid.org/0000-0002-2905-7524

i Hyung Kim

https://orcid.org/0000-0001-9821-387X

Conflict of Interest

Received: July 25, 2019

Accepted: October 4, 2019
Amur catfish (or Korean catfish, Silurus asotus) belonging to Siluridae, is widely distributed throughout East Asia including Korea, and is a commercially important freshwater fish in Korean aquaculture [1]. Up to recent, several bacterial pathogens such as Aeromonas hydrophila, A. veronii, Edwardsiella ictaluri, and Vibrio ordalii have been reported to be implemented in the mass mortality of the cultured Amur catfish in Korea [1,2].

Aeromonas spp. are ubiquitous gram-negative bacilli found in various aquatic environments, with several species identified as pathogens in aquatic animals, including fish, reptiles, and amphibians [3]. Among the major fishpathogenic Aeromonas species, A. veronii is associated with Epizootic Ulcerative Syndrome (EUS) and Motile Aeromonas Septicemia (MAS) of fish, with disease outbreaks caused by the bacterium increasing in the recent years [4]. In Korea, several disease outbreaks associated with $A$. veronii have been reported in cultured Israeli carp (Cyprinus carpio) [5,6], the Japanese eel (Anguilla japonica) [7,8], and the Amur catfish [2]. In addition, the emergence of Aeromonas strains resistant to commercial antibiotics commonly used in aquaculture, has raised serious worldwide concerns. The acquisition of resistance to tetracycline and quinolones in fish-pathogenic Aeromonas spp., has confirmed in Korean aquaculture $[9,10]$. Although A. veronii isolated from Korean environmental water already possessed tetracycline resistance [10], the resistance mechanisms in fish-pathogenic isolates remain uninvestigated.

Herein, we report a case of mass mortality in commercially cultured Amur catfish ( $S$. asotus) caused by the A. veronii. From the isolate, the acquisition of tetracycline resistance was verified, and the resistance-related genetic determinants identified. This is the first report of the occurrence of tetracyclineresistant $A$. veronii infection related to mass mortality in commercially cultured Amur catfish in Korea.

In March 2019, mass mortality in catfish occurred on a private catfish farm located in Jeollabuk-do Province, Republic of Korea. Prior to the onset of the disease, a batch of catfish in juvenile stage (14 days after hatching) were transferred to a culture tank and maintained at $20 \pm 2^{\circ} \mathrm{C}$. The water quality 
Hyo Yeon Kim, Jin Woo Jun, Se Ra Lim, Seon Young Park, Jee Eun Han, Se Chang Park, Ji Hyung Kim

parameters including dissolved oxygen, $\mathrm{pH}$, and ammonia were appropriately maintained. The onset of disease symptoms was first observed four days post transfer into the culture tank, while cumulative mortality reported around six days after the transfer. The symptoms were characterized by the presence of severe, open dermal ulcers with focal hemorrhages on the head, middle of the body, and the dorsal regions of the fish, similar to the typical symptoms in EUS [4]. Dead or moribund fish (average body length $77.0 \pm 2.6$ $\mathrm{mm}$, average body weight $3.7 \pm 0.5 \mathrm{~g}$ ) were submitted to the Infectious Diseases Research Center, Korea Research Institute of Bioscience and Biotechnology for investigation of etiological agents.

During the post-mortem analysis, several deep ulcerations that extended through muscle tissue were reported, and a weak accumulation of ascitic fluid in the peritoneal cavities was observed (Fig. 1). Parasitological examinations did not reveal the presence of external or gill parasites on the catfish. Sterile swabs from kidney and skin ulcers were streaked onto 5\% sheep blood agar plates (BAPs) (Synergy innovation, Korea) and incubated at $20^{\circ} \mathrm{C}$ for $24 \mathrm{~h}$. Similar $\beta$-hemolytic bacterial growth was observed in plates streaked with kidney and skin ulcer swabs. The colonies were repeatedly sub-cultured onto BAPs until pure isolates were obtained, and the isolated $\beta$-hemolytic bacteria were identified using

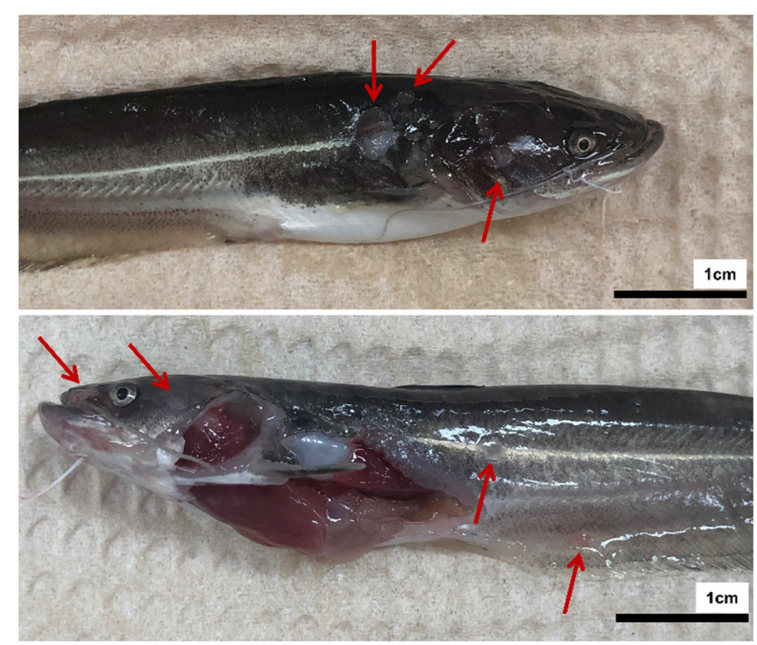

Fig. 1. Aeromonas veronii infected juvenile Amur catfish (Silurus asotus) showing severe, open dermal ulcerations (red arrows) on the head, on the middle of the body, and on the dorsal regions of the fish. Scale bars indicate one centimeter.

Table 1. List of polymerase chain reaction primers used in this study

\begin{tabular}{|c|c|c|}
\hline Primer name & Sequence (5'-3') & Reference \\
\hline \multicolumn{3}{|l|}{ Bacterial identification } \\
\hline \multicolumn{3}{|l|}{ 16s rRNA } \\
\hline $785 \mathrm{~F}$ & GGATTAGATACCCTGGTA & \multirow{2}{*}{ Universal primer } \\
\hline $907 \mathrm{R}$ & CCGTCAATTCMTTTRAGTTT & \\
\hline \multicolumn{3}{|l|}{ rроB } \\
\hline Pasrpob-L & GCAGTGAAAGARTTCTTTGGTTC & \multirow{2}{*}{ [11] } \\
\hline Rpob-R & GTTGCATGTTNGNACCCAT & \\
\hline \multicolumn{3}{|l|}{ Tetracycline resistance } \\
\hline tetA_F & GCTACATCCTGCTTGCCTTC & \multirow{10}{*}{ [9] } \\
\hline tetA_R & GCATAGATCGCCGTGAAGAG & \\
\hline tetB_F & TCATTGCCGATACCACCTCAG & \\
\hline tetB_R & CCAACCATCATGCTATTCCATCC & \\
\hline tetC_F & CTGCTCGCTTCGCTACTTG & \\
\hline tetC_R & GCCTACAATCCATGCCAACC & \\
\hline tetD_F & TGTGCTGTGGATGTTGTATCTC & \\
\hline tetD_R & CAGTGCCGTGCCAATCAG & \\
\hline tetE_F & ATGAACCGCACTGTGATGATG & \\
\hline tetE_R & ACCGACCATTACGCCATCC & \\
\hline \multicolumn{3}{|l|}{ Virulence factors } \\
\hline alt_F & AAAGCGTCTGACAGCGAAGT & \multirow{6}{*}{ [12] } \\
\hline alt_R & AGCGCATAGGCGTTCTCTT & \\
\hline ast_F & ATCGTCAGCGACAGCTTCTT & \\
\hline ast_R & CTCATCCCTTGGCTTGTTTTTAC & \\
\hline aerA/haem_F & CCTATGGCCTGAGCGAGAAG & \\
\hline aerA/haem_R & CCAGTTCCAGTCCCACCACT & \\
\hline
\end{tabular}

rRNA, ribosomal RNA. 
MALDI-TOF MS (Macrogen Inc., Korea) and 16S ribosomal RNA (rRNA) sequencing (Macrogen Inc.). The obtained results indicated that the isolates belong to the genus Aeromonas, but failed to discriminate to the species level. Therefore, the rроB gene of the isolates that encodes the $\beta$-subunit of the DNA-dependent RNA polymerase was amplified and sequenced using the primers Pasrpob-L/Rpob-R [11] (Table 1). The obtained sequences were compared to the type strains of the Aeromonas spp. in the GenBank database. The rpoB sequences were identical between our isolates and most similar to $A$. veronii ATCC $35624^{\mathrm{T}}$ (98.4\% nucleotide identity) among the species in the Aeromonadaceae (Fig. 2). Based on the results of $16 \mathrm{~S}$ rRNA and $r p o B$ comparisons, the $\beta$-hemolytic bacterial isolates were identified as $A$. veronii. Here, the simultaneous isolation of $A$. veronii from the ulcers and kidneys of the catfish clearly indicates that the same bacteria caused MAS and EUS. The kidney-isolated A. veronii strain was designated AVNIH1 and used for further analysis in this study.

Biochemical characteristics of the A. veronii strain AVNIH1 were analyzed using the API 20E system (bioMérieux, France) following the manufacturer's protocol. The result was compared to $A$. veronii strain KC-1109, previously implicated in the mortality of adult catfish cultured in Korea [2], as well as ATCC 9071 and ATCC 35624, and the results are shown in Supplementary Table 1 . The results showed that overall the biochemical phenotype of strain AVNIH1 was very similar to those of strain KC-1109 and ATCC 9071 but differed from ATCC $35624^{\mathrm{T}}$. Based on the positive results of the AMY test (oxidation of amygdalin), our isolate was distinct from strain KC-1109 and ATCC 9071.

To evaluate the pathogenic potential of the A. veronii isolate, the presence of Aeromonas-specific virulence-related genes coding for cytotoxic heat-labile enterotoxin (aer, also known as aerolysin/hemolysin), cytotonic heat-labile entero- toxin (alt), and a cytotonic heat-stable enterotoxin (ast) were determined by polymerase chain reaction (PCR) analyses [12] (Table 1). The isolate only possessed the aer gene, almost identical ( $>99 \%$ amino acid identity) to those from other fish-pathogenic Aeromonas spp., including A. veronii strains X11 (CP024930) and AKEL/SRLAAH/2017 (MH757092). Aerolysin was reported to be one of the major virulent factors in the pathogenesis of $A$. veronii-associated fish diseases, and the aerolysin-mediated aerotaxis has been determined as a causal factor in MAS and EUS [13]. Based on these results, the isolated $A$. veronii strain AVNIH1 demonstrated a strong possibility of causing EUS in this case, and also possessed a pathogenic potential in other fish species.

Antimicrobial susceptibility tests for the $A$. veronii isolate were performed using the disk diffusion method, using a total of 20 antimicrobial agents (Oxoid Ltd., UK), belonging to 9 different classes according to the guidelines of the Clinical and Laboratory Standards Institute [14]. According to the results of the disc diffusion test, the isolate was intermediate to imipenem $(10 \mu \mathrm{g})$ and ciprofloxacin $(10 \mu \mathrm{g})$, and resistant to tetracycline $(30 \mu \mathrm{g})$ (Table 2$)$. The minimum inhibitory concentration (MIC) of $A$. veronii strain AVNIH1 against tetracycline $(256-0.015 \mu \mathrm{g})$ were further determined using MIC Evaluator strip (Oxoid Ltd.), and the MIC value was estimated to be $16 \mu \mathrm{g}$. To determine the genetic determinants associated with resistance to tetracycline, a multiplex PCR assay was conducted as previously described [9] to amplify the five tetracycline resistant genes (tetA to tetE) (Table 1). A total of two PCR amplicons were detected from $A$. veronii strain AVNIH1, and the sequencing analyses revealed that the isolate simultaneously possessed tetA and tetE as its genetic determinants associated with resistance to tetracycline. The newly identified tet $A$ and $t e t E$ in $A$. veronii strain AVNIH1 were identical to those were found in $A$ hydrophila

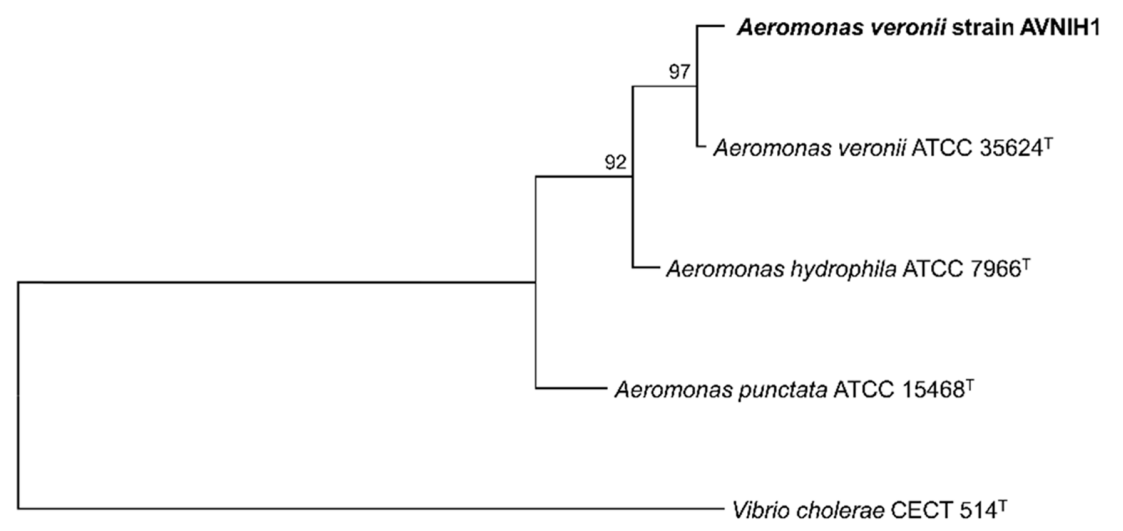

\section{$\longmapsto 0.050$}

Fig. 2. Maximum-likelihood tree based on the nucleotide sequences of rpoB in Aeromonas veronii strain AVNIH1 to some representative type strains of Aeromonas species and the outgroup Vibrio cholerae CECT $514^{\mathrm{T}}$. The scale bar represents 0.05 nucleotide substitutions per site. 
Table 2. Antibiotic resistance profiles of Aeromonas veronii strain AVN1H1

\begin{tabular}{lcc}
\hline \hline \multicolumn{1}{c}{ Antimicrobial agents } & Disk diffusion & MIC $(\mu \mathrm{g} / \mathrm{mL})$ \\
\hline Piperacillin-tazobactam $(110 \mu \mathrm{g})$ & $\mathrm{S}$ & ND \\
Cefepime $(30 \mu \mathrm{g})$ & $\mathrm{S}$ & ND \\
Cefotaxime $(30 \mu \mathrm{g})$ & $\mathrm{S}$ & $\mathrm{ND}$ \\
Ceftazidime $(30 \mu \mathrm{g})$ & $\mathrm{S}$ & $\mathrm{ND}$ \\
Cefuroxime $(30 \mu \mathrm{g})$ & $\mathrm{S}$ & $\mathrm{ND}$ \\
Imipenem $(10 \mu \mathrm{g})$ & $\mathrm{I}$ & $\mathrm{ND}$ \\
Meropenem $(10 \mu \mathrm{g})$ & $\mathrm{S}$ & $\mathrm{ND}$ \\
Aztreonam $(30 \mu \mathrm{g})$ & $\mathrm{S}$ & $\mathrm{ND}$ \\
Amikacin $(30 \mu \mathrm{g})$ & $\mathrm{S}$ & $\mathrm{ND}$ \\
Gentamicin $(10 \mu \mathrm{g})$ & $\mathrm{S}$ & $\mathrm{R}(16)$ \\
Tetracycline $(30 \mu \mathrm{g})$ & $\mathrm{R}$ & $\mathrm{ND}$ \\
Ciprofloxacin $(5 \mu \mathrm{g})$ & $\mathrm{I}$ & $\mathrm{ND}$ \\
Levofloxacin $(5 \mu \mathrm{g})$ & $\mathrm{S}$ & $\mathrm{ND}$ \\
Chloramphenicol $(30 \mu \mathrm{g})$ & $\mathrm{S}$ & $\mathrm{S}$ \\
Trimethoprim-sulfamethoxazole $(25 \mu \mathrm{g})$ & $\mathrm{S}$ & ND
\end{tabular}

ND, not done; MIC, minimum inhibitory concentration.

strain 23-C-23 plasmid (CP038466) and A. veronii strain MS-18-37 chromosome (CP033604), respectively.

Although $A$. veronii infections, which caused epidermal exfoliation and muscular necrosis in cultured adult Amur catfish, have been previously reported in Korea [2], the isolated strain KC-1109 did not demonstrated a resistance to tetracycline, which is commonly used to treat bacterial diseases in Korean aquaculture. However, we were able to detect phenotypical tetracycline resistance and also identify its genetic determinants (tetA and tetE) from A. veronii strain AVNIH1, suggesting that catfish-pathogenic $A$. veronii in Korea have already acquired resistance to the tetracycline. In actuality, the presence of tetracycline resistance genes (tet $A$ ) in $A$. veronii with environmental origin have already been reported [10]; however, simultaneous acquisition of the genetic determinants (tetA and tetE) in the bacteria have not been reported in Korea yet. Of the currently recognized zoonotic Aeromonas species, $A$. veronii has been reported to be implicated in clinical infections in Korea [15]. Therefore, the acquisition of antibiotic-resistance genes in this Aeromonas species may present as a serious potential public health risk, necessitating stricter guidelines for the use of tetracycline to prevent the dissemination and acquisition of tetracycline resistance in Korean aquaculture.

\section{Culture deposition and nucleotide sequence accession} No.

The rpoB, act, tetA, and tetE nucleotide sequences of $A$. veronii strain AVNIH1 have been deposited in the GenBank database under accession numbers MN047444, MN115385, MN047445, and MN047446, respectively. A living axenic culture of $A$. veronii strain AVNIH1 has been deposited in the Korean Culture Center of Microorganisms (KCCM) as KCCM 90343.

\section{Acknowledgments}

This research was supported by the KRIBB Initiative Program and the Collaborative Genome Program of the Korea Institute of Marine Science and Technology Promotion (20180430) funded by the Ministry of Oceans and Fisheries in Republic of Korea.

\section{References}

1. Yu JH, Han JJ, Park KS, Park KH, Park SW. Edwardsiella tarda infection in Korean catfish, Silurus asotus, in a Korean fish farm. Aquacult Res 2009;41:19-26.

2. Kim JD, Do JW, Choi HS, Seo JS, Jung SH, Jo HI, Park M, Lee N, Park SW. Pathological changes in cultured Korean catfish (Silurus asotus) artficially infected with Aeromonas veronii. Korean J Environ Biol 2013;31:486-492.

3. Janda JM, Abbott SL. Evolving concepts regarding the genus Aeromonas: an expanding Panorama of species, disease presentations, and unanswered questions. Clin Infect Dis 1998; 27:332-344.

4. Cai SH, Wu ZH, Jian JC, Lu YS, Tang JF. Characterization of pathogenic Aeromonas veronii bv. veronii associated with ulcerative syndrome from Chinese longsnout catfish (Leiocassis longirostris Günther). Braz J Microbiol 2012;43:382-388.

5. Yi SW, You MJ, Lee HB, Shin GW. A case of Aeromonas veronii infection in Israeli carp (Cyprinus carpio): phylogenetic analysis and antimicrobial resistance. Korean J Vet Serv 2012;35:239-243.

6. Yu JH, Han JJ, Kim HJ, Kang SG, Park SW. First report of Aeromonas veronii infection in farmed Israeli carp Cyprinus carpio in Korea. J Fish Pathol 2010;23:165-176.

7. Joh SJ, Ahn EH, Lee HJ, Shin GW, Kwon JH, Park CG. Bacterial pathogens and flora isolated from farm-cultured eels (Anguilla japonica) and their environmental waters in Korean eel farms. Vet Microbiol 2013;163:190-195. 
8. Yi SW, You MJ, Cho HS, Lee CS, Kwon JK, Shin GW. Molecular characterization of Aeromonas species isolated from farmed eels (Anguilla japonica). Vet Microbiol 2013; 164:195-200.

9. Kim JH, Hwang SY, Son JS, Han JE, Jun JW, Shin SP, Choresca C Jr, Choi YJ, Park YH, Park SC. Molecular characterization of tetracycline- and quinolone-resistant Aeromonas salmonicida isolated in Korea. J Vet Sci 2011; 12:41-48.

10. Han JE, Kim JH, Choresca CH Jr, Shin SP, Jun JW, Chai JY, Park SC. Prevalence of tet gene and complete genome sequencing of tet gene-encoded plasmid (pAHH01) isolated from Aeromonas species in South Korea. J Appl Microbiol 2012;112:631-638.

11. Korczak B, Christensen H, Emler S, Frey J, Kuhnert P. Phylogeny of the family Pasteurellaceae based on rpoB sequences. Int J Syst Evol Microbiol 2004;54:1393-1399.
12. Aravena-Román M, Inglis TJ, Riley TV, Chang BJ. Distribution of 13 virulence genes among clinical and environmental Aeromonas spp. in Western Australia. Eur J Clin Microbiol Infect Dis 2014;33:1889-1895.

13. Foysal MJ, Momtaz F, Ali MH, Siddik MA, Chaklader MR, Rahman MM, Prodhan MS, Cole A. Molecular characterization and interactome analysis of aerolysin (aer) gene from fish pathogen Aeromonas veronii: the pathogenicity inferred from sequence divergence and linked to histidine kinase (cheA). J Fish Dis 2019;42:465-475.

14. Clinical and Laboratory Standards Institute (CLSI). Methods for Antimicrobial Dilution and Disk Susceptibility Testing of Infrequently Isolated or Fastidious Bacteria. 3rd ed. CLSI guideline M45. CLSI, Wayne, 2015.

15. Rhee JY, Jung DS, Peck KR. Clinical and therapeutic implications of Aeromonas bacteremia: 14 years nation-wide experiences in Korea. Infect Chemother 2016;48:274-284.

Supplementary Table 1. Biochemical characteristics of Aeromonas veronii strain AVN1H1 based on the API 20E test

\begin{tabular}{|c|c|c|c|c|}
\hline Strains & A. veronii strain AVNIH1 & A. veronii strain KC-1109 & A. veronii ATCC 9071 & A. veronii ATCC $35624^{\mathrm{T}}$ \\
\hline ONPG & + & + & + & - \\
\hline $\mathrm{ADH}$ & + & + & + & - \\
\hline LDC & - & - & - & + \\
\hline ODC & - & - & - & + \\
\hline CIT & + & + & + & + \\
\hline $\mathrm{H}_{2} \mathrm{~S}$ & - & - & - & - \\
\hline URE & - & - & - & - \\
\hline TDA & - & - & - & - \\
\hline IND & - & - & - & - \\
\hline VP & + & + & + & + \\
\hline GEL & + & + & + & + \\
\hline GLU & + & + & + & + \\
\hline MAN & + & + & + & + \\
\hline INO & - & - & - & - \\
\hline SOR & - & - & - & - \\
\hline RHA & - & - & - & - \\
\hline SAC & + & + & + & + \\
\hline MEL & - & - & - & - \\
\hline AMY & + & - & - & - \\
\hline ARA & - & - & + & - \\
\hline
\end{tabular}

ONPG, $\beta$-galactosidase; ADH, arginine dihydrolase; LDC, lysine decarboxylase; ODC, ornithine decarboxylase; CIT, citrate utilization; $\mathrm{H}_{2} \mathrm{~S}, \mathrm{H}_{2} \mathrm{~S}$ production; URE, urease; TDA, tryptophane deaminase; IND, indole production; VP, Voges-Proskauer; GEL, gelatinase; GLU, glucose; MAN, mannitol; INO, inositol; SOR, sorbitol; RHA, rhamnose; SAC, saccharose; MEL, melibiose; AMY, amygdalin; ARA, arabinose. 